\title{
Reaction Kinetic Form for Lumped Process System Models
}

\author{
Katalin M. Hangos * Gábor Szederkényi * \\ Antonio A. Alonso** \\ * Process Control Research Group, Systems and Control Laboratory, \\ Computer and Automation Research Institute, \\ Hungarian Academy of Sciences \\ P.O. Box 63, H-1518 Budapest, Hungary \\ e-mail:\{hangos,szeder\}@scl.sztaki.hu \\ ** (Bio)Process Engineering Group, IIM-CSIC, Spanish National \\ Research Council, C/Eduardo Cabello, 6, 36208 Vigo, Spain \\ e-mail: antonio@iim.csic.es
}

\begin{abstract}
The class of isotherm lumped process systems with constant overall mass in each of its balance volumes is considered in this paper. It is shown that the convection and transfer mechanisms can be described by a mass action law chemical reaction network (MAL CRN) with only linear kinetics where the in- and outflow terms are represented using the zero complex. This gives rise to a convection and transfer reaction sub-graph that connects atomic complexes consisting of the components in the balance volumes of the system. The real chemical reactions in each of the balance volumes can be described by their own CRNs, where the chemical reaction sub-graphs may be disconnected from the convection and transfer reaction sub-graph when nonlinear reactions occur. In the case of chemical reactions with a finite number of steady state points, the underlying linear dynamical system enables us to construct a dynamically similar linear overall reaction graph for the whole process system. The resulting overall reaction graph can be used for structural stability analysis by applying its graph theoretical properties.
\end{abstract}

Keywords: Process control; Chemical variables control; Modelling; Graph theoretic models; Structural stability;

\section{INTRODUCTION}

Process systems are often highly nonlinear with a wide range of nonlinear phenomena that make their dynamic analysis and control a challenging task. The major sources of the nonlinearity in process systems are the chemical reactions. A separate special positive nonlinear system class, the chemical reaction networks (CRN) with mass action law (MAL) kinetics has been formed to characterize their nonlinear dynamic behavior. It has been shown that the MAL CRN system class is a wide class, that is often used to model complex biological mechanisms [9], or even models of application fields far from chemistry such as mechanical or electrical systems [10]. The increasing interest for this field is shown by numerous surveys and tutorials in different journals [11], [2], ,1].

Motivated by the fact that MAL CRNs exhibit all the qualitative dynamic behavior patterns (e.g. oscillations, chaotic behavior, stable and unstable equilibrium points) that a lumped process system with smooth nonlinearities may show, it is interesting to study the possibility of deriving a MAL CRN representation, i.e. a model in MAL CRN form for them. That would enable to use the strong structural results available in chemical reaction network

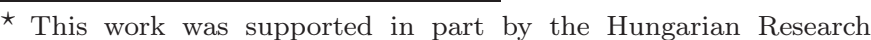
Fund through grant 83440, and by the Control Engineering Research Group of the Budapest University of Technology and Economics.
}

theory for dynamic analysis (stability, controllability, etc.) and controller design of complex nonlinear process systems.

The aim of this paper is to make the first step towards the development of MAL CRN representation of lumped nonlinear process systems in the simplest possible case, when isothermal and isobaric conditions are assumed with constant overall mass in each of the balance volumes. A possible way of using the graph-theoretical properties of the constructed reaction graph of the $\mathrm{CRN}$ representation for structural stability analysis is also demonstrated.

\section{BASIC NOTIONS}

Consider a lumped process system, where each of its balance volumes are perfectly stirred (lumped). Then its dynamic model is in the form of a set of ordinary differential equations (possibly equipped with algebraic equations, but we assume that these can be substituted into the balance equations).

In order to have the simplest possible case, the following general assumptions are made:

(1) constant temperature, i.e. no energy balance equations are considered,

(2) constant pressure (in-compressible fluid phases),

(3) constant physico-chemical properties. 
$N$ lumped balance volumes are considered in the system that are described using the following quantities.

- The convection between balance volumes are described by mass flow rates ${ }^{i j} q$ from balance volume $i$ to $j$, that are measured in units $\mathrm{kg} / \mathrm{s}$.

- The concentration (measured in $\mathrm{mol} / \mathrm{kg}$ ) of component (or specie) $\mathrm{A}_{s}$ in balance volume $j$ is denoted by $\left.{ }^{j} \mathrm{~A}_{s}\right]={ }^{j} c_{s}$.

- The reaction rate constants are denoted by $k_{\ell, l}$, where the pair $\ell, l$ is the identifier of the reaction.

- Mass transfer coefficients (including the transfer area) are used for characterizing the transfer between balance volumes $i$ and $j$, that are denoted by ${ }^{i j} K$, and ${ }^{i j} K={ }^{j i} K$.

\subsection{Dynamic model equations}

Under the general assumptions above, the model equations originate from the overall mass $\left({ }^{i} m\right)$ and component masses $\left({ }^{i} m_{s}={ }^{i} m^{i} c_{s}, s=1, \ldots, L\right)$ balances for each balance volume, respectively. These dynamic balances are of the following general form for lumped balance volumes [7]:

$$
\left\{\begin{array}{l}
\text { rate of } \\
\text { change }
\end{array}\right\}=\left\{\begin{array}{c}
\text { in- } \\
\text { flow }
\end{array}\right\}-\left\{\begin{array}{c}
\text { out- } \\
\text { flow }
\end{array}\right\} \pm\left\{\begin{array}{c}
\text { source } \\
\text { sink }
\end{array}\right\}
$$

The first two terms on the right-hand side of the above equation correspond to the in- and outbound convection terms, while in the source or sink terms may correspond to various other mechanisms. For the sake of simplicity we only assume to have the following most common mechanisms:

- component mass transfer between two balance volumes connected by a joint boundary,

- chemical reactions within any balance volume.

\subsection{Chemical reaction networks and the reaction graph}

A CRN obeying the mass action law is a closed system where chemical species $\mathrm{A}_{s}, s=1, \ldots, L$ take part in $r$ chemical reactions. The concentrations of the species denoted by $c_{s},(s=1, \ldots, L)$ form the state vector $\mathbf{c}$, i.e. $c_{s}=\left[\mathrm{A}_{s}\right]$. The elementary reaction steps have the following form:

$$
\sum_{s=1}^{L} \alpha_{s j} \mathrm{~A}_{s} \rightarrow \sum_{s=1}^{L} \beta_{s l} \mathrm{~A}_{s}
$$

where $\alpha_{s j}$ is the so-called stoichiometric coefficient of component $\mathrm{A}_{s}$ in reaction $C_{j} \rightarrow C_{l}$, and $\beta_{s l}$ is the stoichiometric coefficient of the product $\mathrm{A}_{s}$. The linear combinations of the species in Eq. (2), namely $C_{j}=\sum_{s=1}^{L} \alpha_{s j} \mathrm{~A}_{s}$ and $C_{l}=\sum_{s=1}^{L} \beta_{s l} \mathrm{~A}_{s}$ are called the complexes and are denoted by $\bar{C}_{1}, C_{2}, \ldots, C_{m}$. Reactions may share complexes in complex reaction schemes, therefore $m$ is generally not equal to the number of reactions. Moreover, reactions are assumed to be irreversible in classical reaction kinetic systems, therefore the stoichiometric coefficients are always nonnegative integers.

The reaction rates of the individual reactions $C_{j} \longrightarrow C_{l}$ can be described as

$$
\rho_{j l}(\mathbf{c})=k_{j, l} \prod_{s=1}^{L}\left[\mathrm{~A}_{s}\right]^{\alpha_{s j}}=k_{j, l} \prod_{s=1}^{L} c_{s}^{\alpha_{s j}}
$$

where $k_{j, l}>0$ is the reaction rate coefficient of the reaction.

In our computations, the following form will be used for the description of the dynamics of CRNs obeying the mass action law [5]:

$$
\dot{\mathbf{c}}=Y \cdot A_{k} \cdot \varphi(\mathbf{c})
$$

where $\alpha_{s j}=Y_{s j}, Y \in \mathbb{R}^{L \times m}$ stores the stoichiometric composition of the complexes, $A_{k} \in \mathbb{R}^{m \times m}$ contains information about the structure of the reaction network, and $\varphi: \mathbb{R}^{L} \mapsto \mathbb{R}^{m}$ is a monomial-type vector mapping given by

$$
\varphi_{j}(\mathbf{c})=\prod_{s=1}^{L} c_{s}^{\alpha_{s j}}, \quad j=1, \ldots, m
$$

$A_{k}$ is a column conservation matrix (i.e. the sum of the elements in each column is zero), called the Kirchhoff matrix of the CRN, defined as

$$
A_{k, l j}=\left\{\begin{array}{cl}
-\sum_{\substack{\ell=1 \\
\ell \neq j \\
k_{j l},}}^{m} k_{l, \ell}, & \text { if } l=j
\end{array}\right.
$$

It is important to note that the pair $\left(Y, A_{k}\right)$ uniquely characterizes a particular $\mathrm{CRN}$ with its structure and parameters.

To handle the exchange of materials between the environment and the reaction network, the so-called "zerocomplex" can be introduced and used which is a special complex where all stoichiometric coefficients are zero i.e., it is represented by a zero column vector in the $Y$ matrix [5]. Note, however, that the presence of the zero complex may imply the openness of the reaction kinetic system.

Similarly to [5] and many other authors, the following weighted directed graph (called reaction graph) is assigned to the reaction network (2). The directed graph $D=$ $\left(V_{d}, E_{d}\right)$ of a reaction network consists of a finite nonempty set $V_{d}$ of vertices and a finite set $E_{d}$ of ordered pairs of distinct vertices called directed edges. The vertices correspond to the complexes, i.e. $V_{d}=\left\{C_{1}, C_{2}, \ldots C_{m}\right\}$, while the directed edges represent the reactions, i.e. $\left(C_{l}, C_{j}\right) \in$ $E_{d}$ if complex $C_{l}$ is transformed to $C_{j}$ in the reaction network. The reaction rate coefficients $k_{l, j}$ for $j=1, \ldots, m$ in (3) are assigned as positive weights to the corresponding directed edges in the graph.

An example of a reaction graph is seen in Fig. 2.

\subsection{Simple example}

In order to illustrate the constructions, the following simple nonlinear example will be used.

The process system consists of a reactor and a separator shown in Fig. 1.

In the reactor we consider a set of chemical reactions 


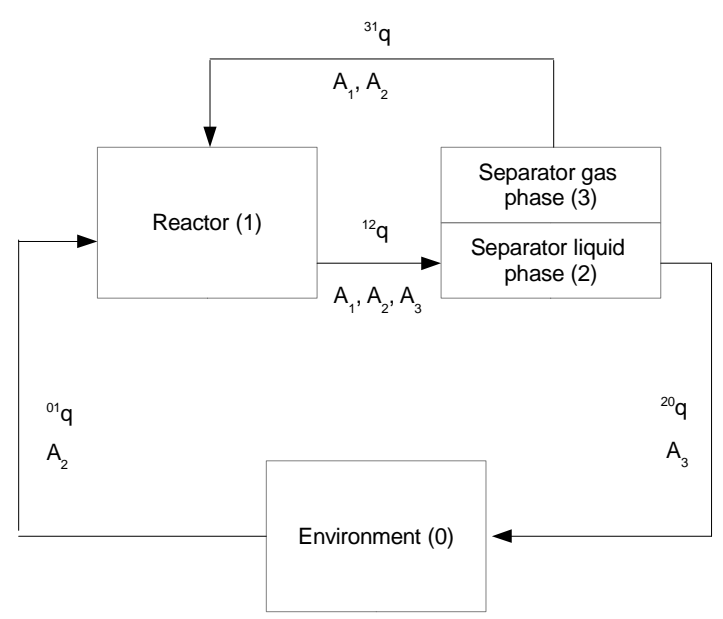

Fig. 1. The flowsheet of the process example

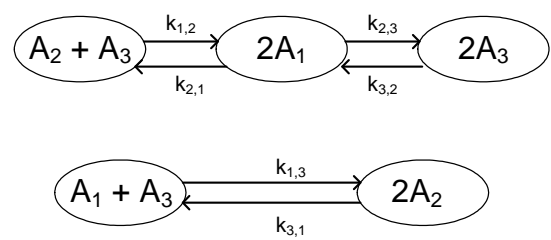

Fig. 2. The simple reaction graph

$$
\begin{aligned}
& \mathrm{A}_{2}+\mathrm{A}_{3} \underset{k_{2,1}}{\stackrel{k_{1,2}}{\rightleftharpoons}} 2 \mathrm{~A}_{1}, \mathrm{~A}_{1}+\mathrm{A}_{3} \underset{k_{3,1}}{\stackrel{k_{1,3}}{\rightleftharpoons}} 2 \mathrm{~A}_{2} \\
& 2 \mathrm{~A}_{1} \underset{k_{3,2}}{\stackrel{k_{2,3}}{\rightleftharpoons}} 2 \mathrm{~A}_{3}
\end{aligned}
$$

The reaction graph of the above chemical reaction network is depicted in Fig. 2. It is assumed that only component $\mathrm{A}_{2}$ enters the system from the environment, and only component $A_{3}$ leaves it. The separator is ideal in the sense that it separates $A_{1}$ and $A_{2}$ to the gas phase, where $A_{3}$ is not present.

\section{THE CONVECTIVE AND TRANSFER NETWORK}

Besides chemical reactions, mass and component mass convection and transfer are the major mechanisms in an isothermal process system [7]. These are both linear mechanisms in the simplest case, therefore an equivalent linear chemical reaction network $(\mathrm{CRN})$ model is expected.

\subsection{Mass convection and transfer terms for a single balance volume}

Further we assume that the overall mass ${ }^{i} m$ of each balance volume $(i=1, \ldots, N)$ is constant. This implies

$$
\sum_{j=0}^{N}{ }^{j i} q=\sum_{j=0}^{N}{ }^{i j} q
$$

The component mass conservation equations can be written separately for each component (no cross-effects between the convection-transfer processes of the individual components) with concentration ${ }^{i} c$ in the $i$ th balance volume in the following form

$$
{ }^{i} m \frac{d\left({ }^{i} c\right)}{d t}=\sum_{j=0}^{N}{ }^{j i} q^{j} c-\sum_{j=0}^{N}{ }^{i j} q^{i} c+\sum_{j=0}^{N}{ }^{j i} K\left({ }^{j} c-{ }^{i} c\right)+{ }^{i} m^{i} R
$$

where ${ }^{j i} K$ is the mass transfer coefficient and ${ }^{i} R$ is the overall reaction rate for the component. The mass transfer coefficients obey the identity ${ }^{j i} K={ }^{j i} K$

The reaction-kinetic analogue form of the above conservation equation is then obtained in the following form

$$
\begin{aligned}
\frac{d^{i} c}{d t}= & \sum_{j=0}^{N}\left[\left(\frac{{ }^{j i} q}{{ }^{i} m}+\frac{{ }^{j i} K}{{ }^{i} m}\right) \cdot{ }^{j} c\right]- \\
& -\left[\sum_{j=0}^{N}\left(\frac{{ }^{i j} q}{{ }^{i} m}+\frac{{ }^{j i} K}{{ }^{i} m}\right)\right] \cdot{ }^{i} c+{ }^{i} R
\end{aligned}
$$

Model properties Consider the case when no reaction occurs, i.e. ${ }^{i} R=0$. Then the equations (10) have the following properties.

(1) The equations correspond to an open system model, since the summation starts at $j=0$, therefore the convection and transfer to and from the environment (the balance volume with index 0) are also taken into account.

(2) No balances are written for the environment, and the concentration of any component in the environment ${ }^{0} c$ is considered as a constant.

(3) The above model is linear and of reaction kinetic type, since the coefficient of ${ }^{i} c$ is negative, while the cross-effect terms are always positive.

(4) One can construct dynamic equations of the form of Eq. (10) separately for each component, i.e. for ${ }^{i} c \in$ $\left\{{ }^{i} c_{1}, \ldots,{ }^{i} c_{L}\right\}$ in the balance volume $i$ with the same coefficients but with a different inlet concentrations ${ }^{j} c, j=0, \ldots, N$, since only the chemical reactions can transform the components to each other.

\subsection{Connecting balance volumes}

In order to investigate the reaction kinetic model of the convection and transfer network only, we assume that no chemical reaction is taking place. Since the dynamic equations for the components are independent of each other (see property (4) above), we only consider a general component with concentration ${ }^{i} c$ in the $i$ th balance volume when developing the overall model of the convection and transfer network.

Let us denote the combined coefficient of convection and transfer from the $j$ th balance volume to the $i$ th balance volume by ${ }^{j i} Q$

$$
{ }^{j i} Q=\left({ }^{j i} q+{ }^{j i} K\right)
$$

with ${ }^{j i} K={ }^{i j} K$. Then the overall dynamic model of the convection and transfer network is the following set of linear in-homogeneous ordinary differential equations (ODEs)

$$
\frac{d^{i} c}{d t}=\sum_{j=0}^{N}\left[\frac{{ }^{j i} Q}{{ }^{i} m} \cdot{ }^{j} c\right]-\left[\sum_{j=0}^{N} \frac{{ }^{i j} Q}{{ }^{i} m}\right] \cdot{ }^{i} c, i=1, \ldots, N
$$


where ${ }^{0} c$ is constant.

3.3 The reaction kinetic model of the convective and transfer network

The reaction graph of a single component The reaction kinetic model of the convective and transfer network of a given component will be constructed from the ODE model (12) in the form of $\frac{d x}{d t}=Y A_{k} \varphi(x)$, where $x$ is the component concentration vector of the $\mathrm{CRN}, Y$ is the stoichiometric matrix, $A_{k}$ is the Kirchoff-matrix, and $\varphi(x)$ is the vector of the reaction monomials.

Because the system is not closed, we need to use the zero complex that corresponds to the environment. Then we have $N+1$ pseudo-components in the systems that form the set of complexes, such that

$$
\begin{gathered}
x=\left[{ }^{1} c, \ldots,{ }^{N} c, 1\right]^{T} ; \varphi_{i}(x)=x_{i}, i=1, \ldots, N+1 \\
Y=\left[\begin{array}{c|c}
I & 0 \\
--+ & -- \\
0 & 0
\end{array}\right]
\end{gathered}
$$

where $I$ is an $N \times N$ unit matrix and $Y \in \mathbb{R}^{N+1 \times N+1}$.

We can construct the upper block of the Kirchhoff matrix $A_{k}$ from Eq. (12)

$$
A=\left[\begin{array}{ccc|c}
-\sum_{j=0}^{N} \frac{{ }^{1 j} Q}{{ }^{1} m} & \ldots & \frac{{ }^{N 1} Q}{{ }^{1} m} & \frac{{ }^{1} Q}{{ }^{0} m} \cdot{ }^{0} c \\
\ldots & \ldots & \ldots & \ldots \\
\frac{{ }^{1} Q}{{ }^{N} m} & \ldots & -\sum_{j=0}^{N} \frac{{ }^{N j} Q}{{ }^{N} m} & \frac{{ }^{0 N} Q}{{ }^{N} m} \cdot{ }^{0} c
\end{array}\right]
$$

The last row in $A_{k}$ with elements $a_{1}, \ldots, a_{N+1}$ can be used to ensure the column conservation property, such that

$$
\tilde{a}_{i}=\left(\sum_{j=0}^{N} \frac{{ }^{i j} Q}{{ }^{i} m}\right)-\left(\sum_{j=1}^{N} \frac{{ }^{i j} Q}{{ }^{j} m}\right)
$$

The actual value of the $i$ th last row element depend on the sign of $\tilde{a}_{i}$ above:

- if $\tilde{a}_{i} \geq 0$ then $a_{i}=\tilde{a}_{i}$ (this induces a pseudo convection-reaction to the environment from the $i$ th balance volume),

- if $\tilde{a}_{i}<0$ then $a_{i}=0$ and $A_{k, i i}=A_{i i}-\tilde{a}_{i}$, that means a pseudo-convection reaction from the environment to the balance volume.

The overall reaction graph Because both the convection and the transfer of any component depends only on the concentration of this component in a certain balance volume, therefore the sub-graph in the reaction graph that describes its convection and transfer network are disjoint from the sub-graphs belonging to any other component, except for the pseudo-component that corresponds to the zero complex, that corresponds to the environment.

The structure of the graph is then partitioned into subgraphs $G_{i}$, where the nodes in $G_{i}$ correspond to the concentration of the $i$ th component in the balance volumes of the process system, i.e. $V_{G_{i}}=\left\{{ }^{j} c_{i} \mid j=1, \ldots, N\right\}$ The zero complex that corresponds to the environment connects these sub-graphs together.
3.4 The CRN representation of the convective and transfer network in the simple example

The reaction graph of the convective and transfer network in the simple example without the pseudo convectionreactions to and from the environment is seen in Fig. 3 . The sub-graphs $G_{i}, i=1,2,3$ of the components $\mathrm{A}_{i}, i=1,2,3$ are also indicated by rectangles.

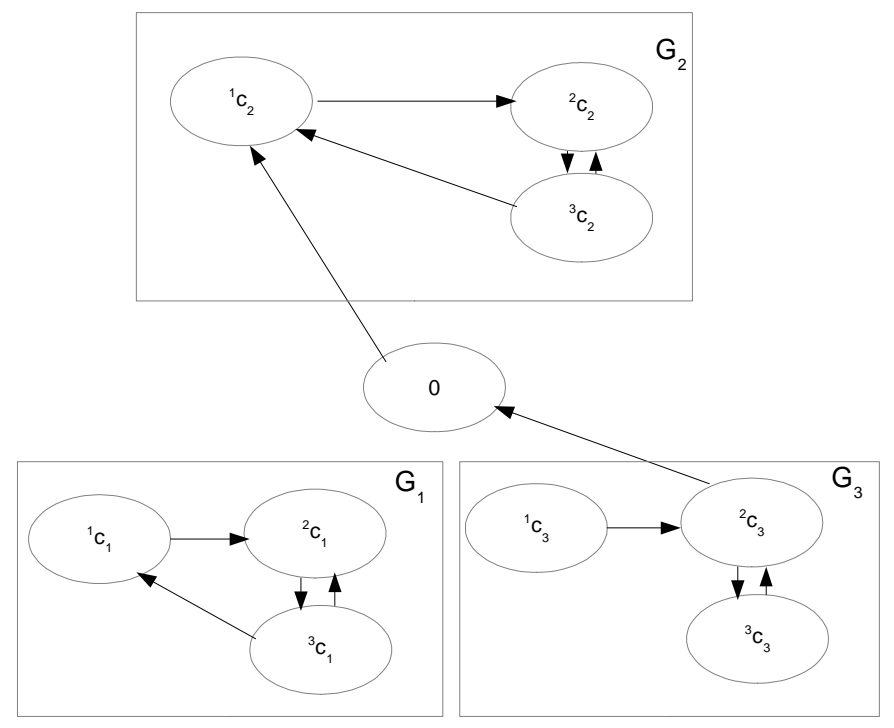

Fig. 3. The reaction graph of the convection and transfer network in the simple example

\section{THE CHEMICAL REACTION NETWORK}

We consider CRNs with mass action type kinetics, that are taking place in each of the balance volumes of the process system. The structure of these CRNs can be conveniently described by reaction graphs (we shall call them chemical reaction graphs), that will not be connected to each other and may not be connected to the convection and transfer reaction graph.

\subsection{The reaction graph of the CRNs}

The components and complexes of a chemical reaction network that belong to the $i$ th balance volume (denoted by ${ }^{i} \mathrm{~A}_{s}, s=1, \ldots, L$ and $\left.{ }^{i} C_{l}, l=1, \ldots, m\right)$ are all different from the components and complexes of any other balance volume, because convection or transfer is needed to "produce" ${ }^{i} \mathrm{~A}_{s}$ from ${ }^{j} \mathrm{~A}_{s}$ (or vice versa) that are described as linear pseudo-reactions in our framework. This implies, that any pair of the chemical reaction graphs ${ }^{i} D_{G}$ and ${ }^{j} D_{G}$ of the CRNs are vertex and edge disjoint. These chemical reaction graphs are the reaction sub-graphs of the overall reaction graph of the process system.

Furthermore, there exists a joint vertex between the overall convection and transfer network and any of the chemical reaction graphs ${ }^{i} D_{G}$ if there exists a linear reaction in the corresponding $\mathrm{CRN}$, i.e. a complex ${ }^{i} C_{l}$ such that ${ }^{i} C_{l}={ }^{i} \mathrm{~A}_{s}$ for some $s$ and $l$. Otherwise, the chemical reaction graph ${ }^{i} D_{G}$ will be vertex and edge disjoint from any other reaction sub-graphs of the system, and therefore it will be a connected component in itself. 
The overall reaction graph of the simple example As the chemical reactions considered in the simple example are all nonlinear, the overall reaction graph of this process system is a union of two disjoint sub-graphs

- the chemical reaction graph shown in Fig. 2, and

- the convection and transfer reaction graph depicted in Fig. 3.

The reaction graphs with non-connected sub-graphs do not exhibit the key structural dynamical properties of the overall system (for example its structural controllability or observability), and require to combine the structural analysis of the graph (for example to detect if it is reversible) with algebraic analysis of the characterizing matrices $A_{k}$ and $Y$ (for computing the deficiency). Therefore, we construct the structure graph of a dynamically similar linear CRN instead.

\subsection{The underlying dynamically similar linear $C R N$}

First we recall that the convective and transfer network of any isotherm process system is a classical linear compartmental system and thus it is equivalent to a linear CRN, where the complexes, i.e. the nodes of the reaction graph, are the components $\mathrm{A}_{i}$ in a balance volume. Then, there will be any joint complex between the reaction graph of the chemical reaction and that of the convection and transfer network, if there are linear chemical reactions taking place in that balance volume.

In order to overcome this difficulty and to be able to join the reaction graphs originating from the chemical reaction and the convection and transfer network, we will replace the original nonlinear CRN with its dynamically similar linear $C R N$ [8]. The following additional assumptions are needed for this purpose.

(1) The original nonlinear CRN has a single equilibrium point within each stoichiometric compatibility class in the positive orthant.

(2) The number of complexes $m$ is greater than the number of components $L(m \geq L)$, and the stoichiometric matrix $Y$ is of full rank.

Constructing the dynamically similar linear CRN Under the above conditions on a nonlinear CRN, its dynamically similar linear CRN can be constructed as follows [8].

First we decompose $Y^{T} \in \mathbb{R}^{m \times L}$ by possibly permuting its rows to get its block-matrix form

$$
Y^{T}=\left[\begin{array}{c}
Y^{* T} \\
---- \\
N_{Y}^{T} Y^{* T}
\end{array}\right]
$$

where $Y^{*}$ is a square $(L \times L)$ invertible matrix.

Then we decompose the rectangular matrix $Y A_{k}$ into two blocks as $Y A_{k}=\left[W_{x} \mid W_{+}\right]$. Then the square $(L \times L)$ matrix

$$
M_{Y}^{*}=Y^{* T}\left(W_{x}+W_{+} N_{Y}^{T}\right)
$$

determines the reduced linear mapping. Note that this matrix is not of full rank, because its second factor, $\left(W_{x}+\right.$ $W_{+} N_{Y}^{T}$ ) is rank-deficient.
The above construction gives rise to the construction of a dynamically similar $L$ dimensional linear CRN with the reduced stoichiometric matrix $\tilde{Y}=I$ and $\tilde{A}_{k}=M_{Y}^{*}$ in Eq. (18).

\subsection{The linear CRN representation of the simple example}

The reaction graph of the dynamically similar linear chemical reaction network of the nonlinear CRN in Fig. 7 is depicted in Fig. 4. The details of the construction can be

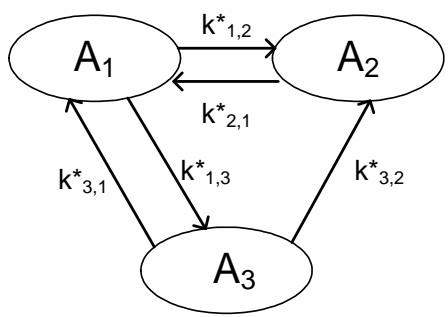

Fig. 4. The reaction graph of the dynamically similar linear $\mathrm{CRN}$

found in [8].

\section{THE CONNECTED CONVECTIVE-TRANSFER AND CHEMICAL REACTION NETWORK AND ITS USE FOR STABILITY ANALYSIS}

\subsection{The connection method}

The overall reaction graph of an isotherm lumped process system with constant overall masses in its balance volumes are constructed in the following steps.

(1) Construct the convection and transfer network of each component over each of the balance volumes in the system: this results in a reaction graph with singlecomponent complexes.

(2) Construct the dynamically similar linear CRN for each of the chemical reaction systems within its respective balance volume and draw its reaction graph. This will also be a reaction graph with singlecomponent complexes.

(3) Join the above obtained graphs along their joint single-component complexes. This will increase the connectivity of the overall reaction graph, that will possibly lead to a connected weakly reversible (deficiency zero) reaction graph proving the global structural stability of the original process system.

\subsection{Structural stability analysis}

Given the overall reaction graph of the connected convective-transfer and reaction network, one can use the celebrated Deficiency Zero theorem [6] for structural stability analysis. This states that for a weakly reversible $M A L C R N$ of deficiency zero - but regardless of the positive values the reaction rate coefficients take - the differential equations of the corresponding reaction system have the following properties: There exists within each positive stoichiometric compatibility class precisely one steady state; that steady state is asymptotically stable; and there is no nontrivial 
cyclic composition trajectory along which all species concentrations are positive.

In the case of the overall reaction graph of the connected convective-transfer and reaction network, one has an underlying linear CRN to analyze, where the number of complexes $\bar{m}$ is equal to the number of pseudo-species $\bar{L}=\bar{m}$. Therefore, the CRN is deficiency zero, if the reaction graph is connected, i.e. it has a single linkage class. Furthermore, the CRN is weakly reversible, if whenever there exists a directed path from complex $C_{i}$ to $C_{j}$ then there also exists a directed path from $C_{j}$ to $C_{i}$. Therefore, structural stability (i.e. stability for each possible reaction rate coefficient value) of the system holds for connected weakly reversible reaction graphs.

\subsection{The reaction kinetic representation and structural stability of the simple example}

The overall reaction graph of the simple example is depicted in Fig. 5. The solid lines represent the convection

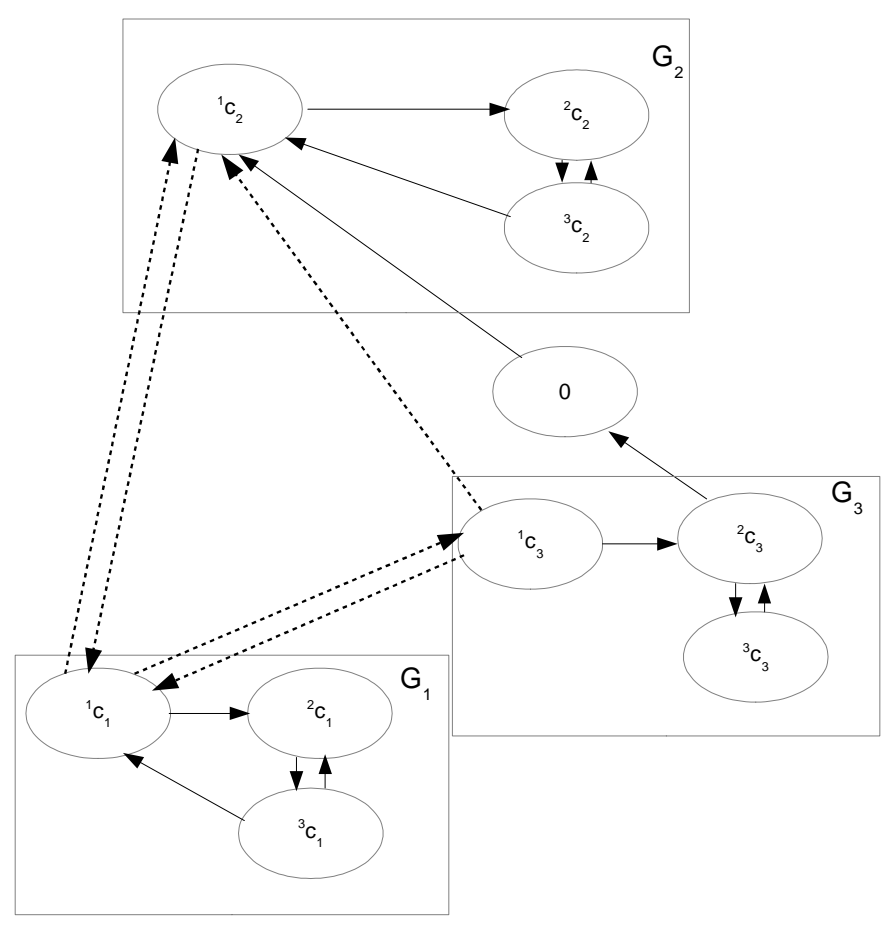

Fig. 5. The reaction graph of the simple example

and transfer edges, while the dashed lines originate from the edges of the reaction graph of the dynamically similar linear CRN.

The overall graph is clearly connected and weakly reversible, therefore the underlying process system is structurally stable.

\section{CONCLUSION}

The class of isotherm lumped process systems with constant overall mass in each of its balance volumes is considered in this paper. It is shown that the convection and transfer mechanisms can be described by a mass action law chemical reaction network (MAL CRN) with only linear kinetics where the in- and outflow terms are represented using the zero complex. This gives rise to a reaction subgraph connecting atomic complexes consisting of the components in the balance volumes of the system.

The real chemical reactions in each of the balance volumes can be described by their own CRNs, where the reaction sub-graphs may be disconnected from the convectiontransfer reaction sub-graph in the case of nonlinear reactions. In the case of chemical reactions with a finite number of steady state points within each stoichiometric compatibility class, the underlying dynamically similar linear system enables us to construct a linear overall CRN for the whole process system.

Finally, the structural stability of the process system can be shown using the deficiency zero theorem for this case, if the overall reaction graph of the connected convectivetransfer and reaction network is connected and weakly reversible.

\section{REFERENCES}

[1] D. Angeli. A tutorial on chemical network dynamics. European Journal of Control, 15:398-406, 2009.

[2] V. Chellaboina, S. P. Bhat, W. M. Haddad, and D. S. Bernstein. Modeling and analysis of mass-action kinetics - nonnegativity, realizability, reducibility, and semistability. IEEE Control Systems Magazine, 29:6078, 2009.

[3] P. Érdi and J. Tóth. Mathematical Models of Chemical Reactions. Theory and Applications of Deterministic and Stochastic Models. Manchester University Press, Princeton University Press, 1989

[4] M. Feinberg. On chemical kinetics of a certain class. Arch. Rational Mech. Anal., 46:1-41, 1972.

[5] M. Feinberg. Lectures on chemical reaction networks. Notes of lectures given at the Mathematics Research Center, University of Wisconsin, 1979.

[6] M. Feinberg. Chemical reaction network structure and the stability of complex isothermal reactors I. The deficiency zero and deficiency one theorems. Chemical Engineering Science, 42:2229-2268, 1972.

[7] K. M.Hangos and I. T. Cameron. Process modelling and model analysis. Academic Press, 2001.

[8] K. M. Hangos and G. Szederkényi. The underlying linear dynamics of some positive polynomial systems. Physics Letters A, 376:3129-3134, 2012.

[9] G. Nicolis and I. Prigogine. Self-organization in Nonequilibrium Systems: From Dissipative Structures to Order through Fluctuations. John Wiley and Sons, New York, 1977.

[10] N. Samardzija, L. D. Greller, and E. Wassermann. Nonlinear chemical kinetic schemes derived from mechanical and electrical dynamical systems. Journal of Chemical Physics, 90 (4):2296-2304, 1989.

[11] E. Sontag. Structure and stability of certain chemical networks and applications to the kinetic proofreading model of T-cell receptor signal transduction. IEEE Trans. Autom. Control, 46:1028-1047, 2001. 